\title{
Transport of Mars-Crossing Asteroids from the Quasi-Hilda Region
}

\author{
Michael Dellnitz, ${ }^{1}$ Oliver Junge, ${ }^{1}$ Martin W. Lo, ${ }^{2}$ Jerrold E. Marsden, ${ }^{3}$ Kathrin Padberg, ${ }^{1}$ \\ Robert Preis, ${ }^{1}$ Shane D. Ross, ${ }^{4}$ and Bianca Thiere ${ }^{1}$ \\ ${ }^{1}$ Faculty of Computer Science, Electrical Engineering and Mathematics, University of Paderborn, D-33095 Paderborn, Germany \\ ${ }^{2}$ Navigation and Mission Design, Jet Propulsion Laboratory, California Institute of Technology, M/S 301-140L, \\ 4800 Oak Grove Drive, Pasadena, California 91109, USA \\ ${ }^{3}$ Control and Dynamical Systems, California Institute of Technology, MC 107-81, Pasadena, California 91125, USA \\ ${ }^{4}$ Department of Aerospace and Mechanical Engineering, University of Southern California, Los Angeles, California 90089, USA
} (Received 30 September 2004; published 16 June 2005)

\begin{abstract}
We employ set oriented methods in combination with graph partitioning algorithms to identify key dynamical regions in the Sun-Jupiter-particle three-body system. Transport rates from a region near the 3:2 Hilda resonance into the realm of orbits crossing Mars' orbit are computed. In contrast to common numerical approaches, our technique does not depend on single long term simulations of the underlying model. Thus, our statistical results are particularly reliable since they are not affected by a dynamical behavior which is almost nonergodic (i.e., dominated by strongly almost invariant sets).
\end{abstract}

PACS numbers: 95.10.Ce, 95.10.Fh, 96.30.Ys

Introduction. - There is much interest in the transport of material throughout the solar system [1-3]. In particular, the numerical simulation of delivery of asteroids to the terrestrial planets is among the most difficult yet interesting problems in celestial mechanics. The recent close approaches of some asteroids to Earth highlight the importance of this problem.

The purpose of this Letter is to make use of new methods in computational dynamical systems to compute the average rates of delivery of quasi-Hilda objects to Marscrossing orbits. The results may be viewed as a first step in the application of these computational methods to interesting astrodynamical problems. Similar numerical techniques have previously been successfully applied to molecular problems [4-8].

In principle, the computation of rates of mass transport can be accomplished by large numerical simulations in which the orbits of test particles are propagated in time including as many interactions as possible [3,9]. However, such calculations are computationally demanding and, due to the chaotic nature of the trajectories, it may be difficult to reliably extract from them information about the statistical quantities of interest. In fact, one of the hallmarks of chaotic dynamical systems is the property that a given initial condition may reach distant regions of phase space, but that may take an arbitrary long time. This very phenomenon may lead to inaccuracies in transport computations that are based on (comparatively few) long term simulations since they may not be a representative sample of the global dynamics. For example, it may be that a dynamical system is nearly uncoupled in the sense that it is possible to decompose the phase space into a finite number of subsets such that there is a very small probability that typical trajectories beginning in each subset will leave this subset in a short time. These almost-invariant sets define macroscopic structures preserved by the dynamics. It may be difficult to compute the probability of going from one of these sets to the other by computing a number of long term simulations; yet this sort of probability is often what is of interest.

This "trapping problem" is obviously relevant in Hamiltonian models that exhibit (perturbed) periodic or quasiperiodic behavior. We avoid this problem in our approach since it is based on an appropriate agglomeration of short time simulations for a large number of globally distributed initial conditions. Rather than characterizing dynamical behavior at the level of trajectories, one can view our approach as tracking the evolution of densities under the influence of the underlying dynamics. A global set oriented method has been developed to perform the associated computations $[6,10,11]$. This basic setup allows us to compute transport rates between arbitrary sets of interest in phase space. Moreover, a priori error estimates on the computed quantities are available [12].

Another classical approach for the treatment of questions about transport is a geometrical framework employing phase space structures related to stable and unstable manifolds of certain periodic points, as in [13]. However, as noted in [12], these methods do not yet scale very well for very long time scales and a generalization to higher dimensional systems is difficult (in contrast to our set oriented approach).

The Letter is organized as follows: first we give a short description of the model used, namely, a first return map in the planar circular restricted three-body problem (PCRTBP) with the Sun and Jupiter as the primaries. Second, we identify a key quasiresonance region by decomposing the phase space into almost-invariant sets, and third, we compute the transport rate from this region into a region that contains all the Mars crossings. We conclude with a discussion of future research directions in this area.

The planar circular restricted three-body problem.The PCRTBP Hamiltonian for the motion of a particle (asteroid) in the field of the Sun and Jupiter is 


$$
H=E=\frac{1}{2}\left(p_{x}^{2}+p_{y}^{2}\right)-\left(x p_{y}-y p_{x}\right)-\frac{m_{S}}{r_{S}}-\frac{m_{J}}{r_{J}}-\frac{1}{2} m_{S} m_{J},
$$

where $E$ is the energy, $r_{S}$ and $r_{J}$ are the distances from the particle to the Sun and Jupiter, respectively, and $m_{S}=$ $1-\mu$ and $m_{J}=\mu$ are the normalized masses of the Sun and Jupiter, respectively. The coordinate system rotates about the common center of mass, the Sun and Jupiter are on the $x$ axis at $(-\mu, 0)$ and $(1-\mu, 0)$, respectively, and $(x, y)$ is the position of the particle relative to the positions of the Sun and Jupiter in this frame, with $p_{x}=$ $\dot{x}-y, p_{y}=\dot{y}+x$ as the conjugate momenta. The motion of the test particle takes place on a three-dimensional energy manifold (defined by a particular value of $E$ ) embedded in the four-dimensional phase space with coordinates $(x, y, \dot{x}, \dot{y})$.

We consider the Poincare surface of section (SOS) defined by $y=0, \dot{y}<0$, and the coordinates on the section are $(x, \dot{x})$. The geometrical interpretation is straightforward: we plot the $x$ coordinate and velocity of the test particle at every conjunction with the planet. As a further restriction, we consider only the motion of test particles in the interior realm (strictly speaking, with mean motion greater than Jupiter's). For orbits interior to the planet's, the SOS is crossed every time the test particle is aligned with Sun and Jupiter and is on the opposite side of the Sun from Jupiter, along $x<0$. So our SOS becomes the twodimensional manifold $M$ defined by $y=0, \dot{y}<0, x<0$, reducing the system to an area and orientation preserving map $f: M \rightarrow M$ on a subset $M$ of $\mathbb{R}^{2}$. Figure 1 shows a mixed phase space structure on this SOS.

Mars crossings, Hilda asteroids, and quasi-Hildas.Also shown in Fig. 1 is a line of constant periapse. Each

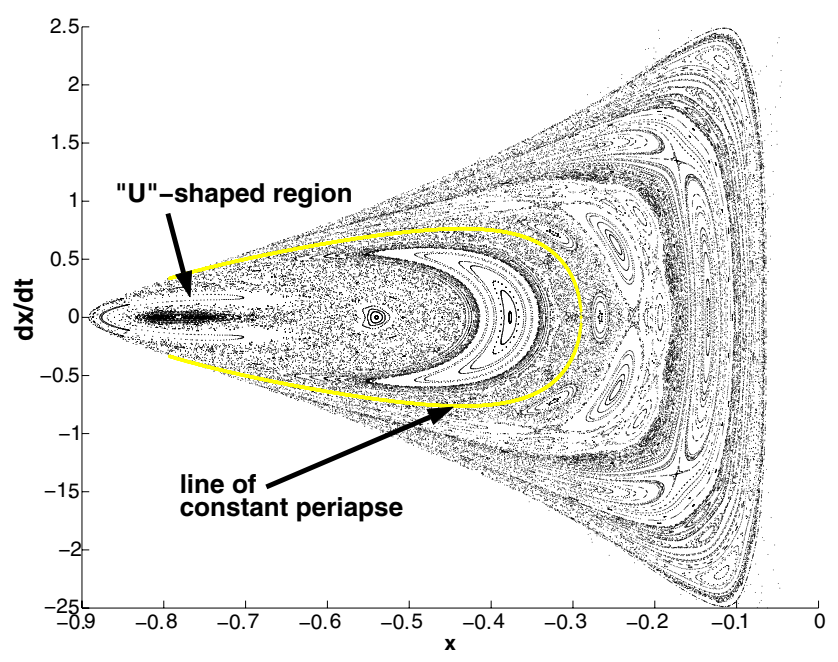

FIG. 1 (color online). The mixed phase space structure of the PCRTBP is shown on this SOS. KAM (Kolmogorov-ArnoldMoser) tori and the chaotic sea are visible. The bright line corresponds to a line of constant periapse. In this case the periapse is equal to the semimajor axis of Mars' orbit around the Sun. $(x, \dot{x}) \in M$ corresponds to an osculating conic orbit about the Sun with an instantaneous semimajor axis $a$ and eccentricity $e$. The line is the locus of points satisfying $r_{p}=$ $a(1-e)$ for a periapse $r_{p}$ equal to the semimajor axis of Mars' orbit around the Sun. Any point in $M$ on this line is an orbit which grazes the orbit of Mars. Keplerian orbits represented by points to the right of this line cross the orbit of Mars.

The energy we consider in this Letter, $E=-1.52$, is just below that of the equilibrium point $L_{1}(E=-1.5199)$, and is a good starting point for understanding dynamics related to the Hilda resonance, the 3:2 resonance with Jupiter. In Fig. 1, the sideways "U"-shaped (or horseshoe-shaped) region on the left indicates this resonance island, which contains the Hilda group of asteroids. The Hilda asteroids owe their longevity to the invariance of this resonance island. However, this island is surrounded by chaotic orbits which give rise to interesting dynamical phenomena that have been noted in previous work. For example, comets known as quasi-Hildas, such as Oterma and Gehrels 3, appear for a time to have Hilda-type orbits until perturbed by Jupiter into a new orbit [14].

In this Letter, we consider quantitatively the phenomenon of transport of quasi-Hildas to the inner solar system. These objects, and their related resonance, exist over a range of energies, but we investigate only one representative energy to elucidate the essential geometry and statistics of this transport. Therefore, we have picked an energy value such that there are no dynamical connections between the interior region and the Jupiter region; i.e., the neck around $L_{1}$ between these two regions is closed. However, the perturbation due to Jupiter can still be significant.

Transport computations. - We consider a situation in which an asteroid (or other body) starts out near the 3:2 mean motion resonance with Jupiter, in a region we call the quasi-Hilda region. This region may contain, e.g., Hilda asteroids which have recently escaped from a stable orbit in the 3:2 resonance island via forces which are not captured by our model. We construct this quasi-Hilda region by computing a global decomposition of the phase space of $f$ into almost-invariant sets. The quasi-Hilda region is then defined as the set from this decomposition which contains the Hilda resonance, as seen in Fig. 2. We are not concerned with how such bodies arrived in this region, but only in their transport to other regions. In particular, we will compute the probability by which these quasi-Hilda objects transit beyond the Mars-crossing line.

Decomposition into almost-invariant sets. - In the following we briefly outline our computational approach. For details we refer to [12].

First, we approximate the recurrent set within $M$ by covering it with a collection of boxes (see Fig. 2). For each box we choose a number of points in the box, perform a forward iteration for each point, and monitor the box reached by the iteration. The transition probability from a 


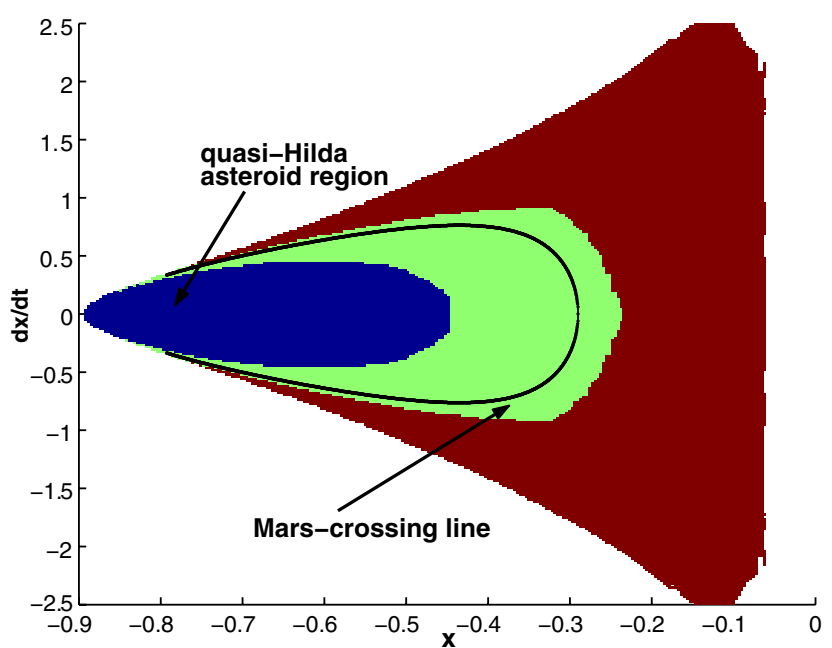

FIG. 2 (color online). A decomposition of a Poincaré SOS into three almost-invariant sets is shown for the mixed regular and chaotic phase space of the restricted three-body problem. The quasi-Hilda region and Mars-crossing line are labeled.

source box to a destination box is measured by the ratio of the number of points from the source box reaching the destination box in one iteration step. We can view this as a graph with the boxes being the vertices and the transition probabilities between the boxes being the weighted, directed edges. We choose the size of the boxes and the number of points such that we achieve an appropriate granularity and accuracy of this transition graph [10].

Second, we partition the vertices of the graph into almost-invariant sets, i.e., into a small number of parts such that each part is highly coupled within itself and only loosely coupled with other parts (see, e.g., $[4,6]$ ). Graph partitioning problems like this one are known to be NP (nondeterministic polynomial time) complete; i.e., no efficient optimal algorithms running in polynomial time are known. For our computations we used an efficient heuristic based on the so-called multilevel paradigm: a large graph is coarsened through several levels to a small graph, the small graph is efficiently partitioned, and the partition is projected back throughout the same levels while being improved on every level. The success of this approach is based on the use of an approximation algorithm for maximum weighted edge matching in the coarsening step and the use of the helpful-set heuristic (providing upper bounds) for the local improvement step. Both algorithms provide provable quality guarantees. The whole concept is described in [15] and implemented in the graph partitioning software library PARTY.

We developed a new tool called GADS (graph algorithms for dynamical systems) [16] which efficiently uses the functionality of the dynamical systems software library GAIO [10], the heuristics from the graph partitioning software library PARTY, and some further implementations of graph algorithms in order to efficiently interlock graph based techniques with set oriented methods.
As one result, we constructed a partition consisting of three almost-invariant sets for the problem considered here (see Fig. 2).

Transport of quasi-Hildas. - The transition graph constructed above may alternatively be viewed as a (sparse) stochastic matrix: each edge in the graph corresponds to a nonzero entry in the matrix and the magnitude of the entry is given by the weight associated to this edge. This transition matrix contains all information for the computation of the transport quantities of interest (see [12]). In our case, we compute the probability that a typical object that starts in the quasi-Hilda region eventually crosses the orbit of Mars, i.e., that a point that starts in the set $R$ in Fig. 3 eventually maps into the set $Q$ under iteration of $f$. Note that in this case the set $Q$ is defined via the Mars-crossing line. The probability for such a transition after $n$ iterations of the map $f$ is given by

$$
p_{R, Q}(n)=\frac{m\left(f^{-n}(Q) \cap R\right)}{m(R)},
$$

where $m$ denotes the volume measure. Let $P=\left(p_{i j}\right), p_{i j}=$ $m\left(f^{-1}\left(B_{i}\right) \cap B_{j}\right) / m\left(B_{j}\right)$, denote the transition matrix (where $B_{1}, \ldots, B_{b}$ are the boxes in the covering). Then the transition probability $p_{R, Q}(n)$ is approximately given by [12]

$$
p_{R, Q}(n) \approx \frac{1}{m(R)} e_{Q}^{T} P^{n} u_{R}
$$

where the $i$ th entry of $e_{Q} \in \mathbb{R}^{b}$ is 1 , if $B_{i} \subset Q$ and 0 otherwise and the $i$ th entry of $u_{R} \in \mathbb{R}^{b}$ is equal to $m\left(B_{i}\right)$ if $B_{i} \subset R$ and 0 otherwise. Figure 4 shows the dependence of the approximated transition probability $p_{R, Q}(n)$ on the number of iterates $n$.

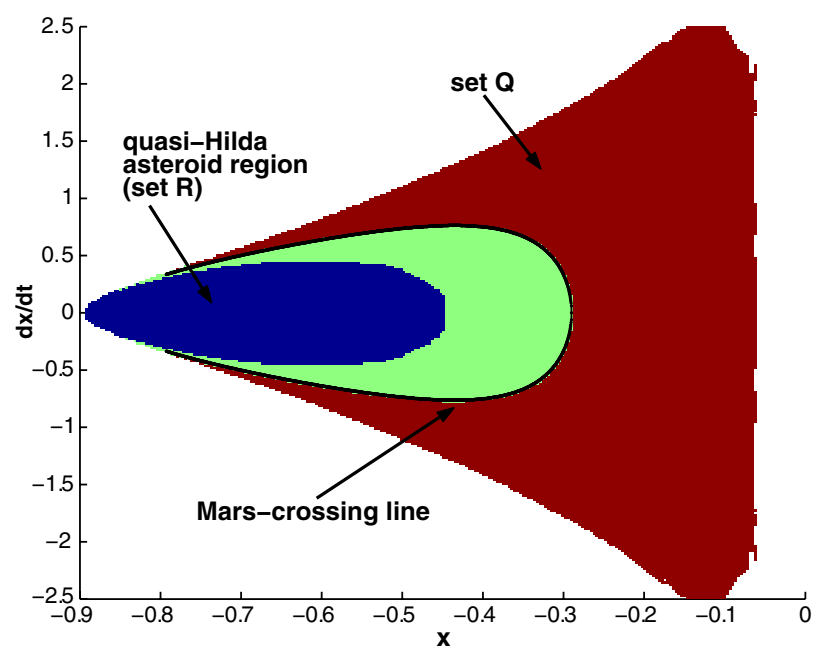

FIG. 3 (color online). Decomposition of the SOS including the quasi-Hilda region $R$ and the region beyond the Mars-crossing line $Q$ as used for the transport computation. 


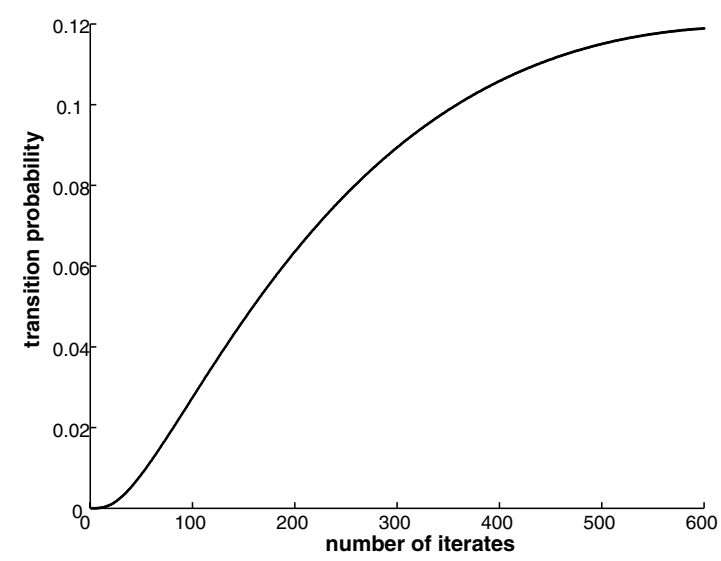

FIG. 4. Transition probability for a particle from the quasiHilda region to the Mars-crossing region as a function of the number of iterates of the return map.

According to this figure, the probability for a typical particle to leave the quasi-Hilda region is around 6\% after 200 iterates of the map, which corresponds to a transit time between 2000 and 6000 Earth years, depending on the location of the particle within the quasi-Hilda region.

The computation of the covering and the associated transition matrix took about $3.5 \mathrm{~h}$ on an Intel Xeon $3.2 \mathrm{GHz}$ processor. We note that the computation of these two objects largely dominates the overall computational cost of our approach.

Future directions. - In the problem studied here, the dynamics was planar for simplicity. Some obvious tasks need to still be carried out, even in this basic context, such as exploring other energy levels as well as model and computational robustness. However, because of the agglomeration of short time simulations for a large number of globally distributed initial conditions in combination with the evolution of densities we believe that this method will be most powerful for systems of higher dimension for which simulations become more difficult and insight into dynamical mechanisms is harder to extract [12]. It may also be useful to combine the methods with time-frequency analysis [17].

This research was partly supported by the DAAD, the DFG Priority Program 1095, the NSF-ITR Grant No. ACI0204932, Grant No. NSF-DMS 0402842, the Max Planck
Society, and the California Institute of Technology President's Fund. This work was carried out in part at the Jet Propulsion Laboratory and California Institute of Technology under a contract with National Aeronautics and Space Administration.

[1] S.A. Astakhov, A.D. Burbanks, S. Wiggins, and D. Farrelly, Nature (London) 423, 264 (2003).

[2] M. Cuk and J. A. Burns, Icarus 167, 369 (2004)

[3] B. J. Gladman, J. A. Burns, M. Duncan, P. Lee, and H. F. Levison, Science 271, 1387 (1996).

[4] M. Dellnitz and R. Preis, Proceedings of SNSC'O1, edited by F. Winkler, LNCS 2630 (Springer, New York, 2003), pp. 183-209

[5] P. Deuflhard, M. Dellnitz, O. Junge, and C. Schütte, in Computational Molecular Dynamics: Challenges, Methods, Ideas, edited by P. Deuflhard, J. Hermans, B. Leimkuhler, A. E. Mark, S. Reich, and R. D. Skeel, Lecture Notes in Computational Science and Engineering Vol. 4 (Springer-Verlag, Berlin, 1998), pp. 98-115.

[6] G. Froyland and M. Dellnitz, SIAM J. Sci. Comput. 24, 1839 (2003).

[7] C. Schütte and W. Huisinga, in Handbook of Numerical Analysis X, edited by P. G. Ciaret and J.-L. Lions (NorthHolland, Amsterdam, 2003).

[8] C. Schütte, J. Walter, C. Hartmann, and W. Huisinga, SIAM Multiscale Modeling and Simulation 2, 501 (2004).

[9] J. Wisdom and M. Holman, Astron. J. 102, 1528 (1991).

[10] M. Dellnitz, G. Froyland, and O. Junge, in Ergodic Theory, Analysis, and Efficient Simulation of Dynamical Systems, edited by B. Fiedler (Springer, New York, 2001), p. 145.

[11] M. Dellnitz and O. Junge SIAM J. Numer. Anal. 36, 491 (1999).

[12] M. Dellnitz, O. Junge, W. S. Koon, F. Lekien, M. W. Lo, J.E. Marsden, K. Padberg, R. Preis, S.D. Ross, and B. Thiere, Int. J. of Bifurcation and Chaos, 15, 699 (2005)

[13] R. S. MacKay, J. D. Meiss, and I. C. Percival, Physica D (Amsterdam) 27, 1 (1987).

[14] C. E. Spratt, J. R. Astron. Soc. Can. 83, 393 (1989).

[15] R. Preis, Ph.D. thesis, University of Paderborn, 2000.

[16] R. Preis, "GADS - Graph Algorithms for Dynamical Systems, User Manual," (to be published).

[17] L. Vela-Arevalo and J.E. Marsden Classical Quantum Gravity 21, S351 (2004). 